\title{
Finite speed of propagation and waiting time for a thin-film Muskat problem
}

\author{
Philippe Laurençot \\ Institut de Mathématiques de Toulouse, UMR 5219, \\ Université de Toulouse, CNRS, 31062 Toulouse CEDEX 9, France \\ (laurenco@math.univ-toulouse.fr)

\section{Bogdan-Vasile Matioc} \\ Institut für Angewandte Mathematik, \\ Leibniz Universität Hannover, Welfengarten 1, 30167 Hannover, \\ Germany (matioc@ifam.uni-hannover.de)
}

(MS received 17 September 2015; accepted 16 February 2016) Propagation at a finite speed is established for non-negative weak solutions to a
thin-film approximation of the two-phase Muskat problem. The expansion rate of the
support matches the scale invariance of the system. Moreover, we determine sufficient conditions on the initial data for the occurrence of waiting time phenomena.

Keywords: finite propagation speed; waiting time; degenerate parabolic system

2010 Mathematics subject classification: Primary 35K65; 35K40; 35B99; 35Q35

\section{Introduction and main results}

The Muskat problem is a complex free-boundary model that was proposed by Muskat [13] to describe the motion of two immiscible fluids with different densities and viscosities in a porous medium with impermeable bottom (such as the intrusion of water into oil). In the limit of thin fluid layers it was shown in [7] that the Muskat problem can be approximated by a strongly coupled parabolic system of equations, which, when neglecting surface tension effects, reads as follows:

$$
\left.\begin{array}{l}
\partial_{t} f=\partial_{x}\left(f \partial_{x}((1+R) f+R g)\right) \\
\partial_{t} g=R_{\mu} \partial_{x}\left(g \partial_{x}(f+g)\right)
\end{array}\right\}
$$

for $(t, x) \in(0, \infty) \times \mathbb{R}$, and is supplemented with initial conditions

$$
f(0)=f_{0}, \quad g(0)=g_{0}, \quad x \in \mathbb{R} .
$$

The constants $R$ and $R_{\mu}$ in $(1.1 a)$, which are assumed in this paper to be positive, are defined as

$$
R:=\frac{\rho_{+}}{\rho_{-}-\rho_{+}} \quad \text { and } \quad R_{\mu}:=\frac{\mu_{-}}{\mu_{+}} R
$$

(C) 2017 The Royal Society of Edinburgh 
with $\rho_{-}$and $\mu_{-}$(respectively, $\rho_{+}$and $\mu_{+}$) denoting the density and viscosity of the lower fluid (respectively, upper fluid). This reduced model retains only the functions $f=f(t, x) \geqslant 0$ and $g=g(t, x) \geqslant 0$ as unknowns, where $f$ is the thickness of the lower fluid layer and $g$ is the thickness of the upper fluid layer, so that $f+g$ is the total height of the fluids. When $R_{\mu}=R$ system $(1.1 a)$ is also a particular case of thin-film models derived in [9] in the context of seawater intrusion.

System $(1.1 a)$ is a degenerate parabolic system with a full diffusion matrix, and can be regarded as a two-phase generalization of the porous medium equation. Among the salient features of the latter are the finite speed of propagation and waiting time phenomena. Recall that the former means that the support of solutions remains compact if it is initially compact, while a waiting time phenomenon refers to the situation in which the solution vanishes at a point on the boundary of the support of its initial condition for some time. Since system $(1.1 a)$ is degenerate and somewhat related to the porous medium equation, it is natural to ask whether these two features also appear in the thin-film Muskat problem. It is the purpose of this paper to provide an affirmative answer to these questions.

There is a huge literature on the finite speed of propagation for degenerate parabolic equations, and various methods have been developed to investigate this issue. In particular, for second-order parabolic equations, e.g. the porous medium equation or the $p$-Laplacian equation, for which the comparison principle is available, this property can be derived by comparison with suitable sub- and supersolutions (see [16] and the references therein). This approach cannot, however, be extended to higher-order equations or to systems, and energy methods have been developed instead (see $[2-4,15]$ and the references therein). These methods were applied, in particular, to the thin-film equation, which is a fourth-order degenerate parabolic equation, and they also work for second-order equations. A few applications to systems of equations can be found in the literature: the finite speed of propagation and the occurrence of waiting time phenomena are shown in [5] for the Poisson-Nernst-Planck system, which is of diagonal type with lower-order coupling, and in [8] for the parabolic-elliptic chemotaxis Keller-Segel system, which one can view as a non-local parabolic equation.

As we shall see below, the energy method is sufficiently flexible to be adapted to study the strongly coupled degenerate parabolic system $(1.1 a)$. Before stating our result, let us introduce the notion of a weak solution to (1.1) to be used hereafter. Let $\mathcal{K}$ denote the positive cone of the Banach space $L_{1}\left(\mathbb{R},\left(1+x^{2}\right) \mathrm{d} x\right) \cap L_{2}(\mathbb{R})$ defined by

$$
\mathcal{K}:=\left\{u \in L^{1}\left(\mathbb{R},\left(1+x^{2}\right) \mathrm{d} x\right) \cap L^{2}(\mathbb{R}): u \geqslant 0\right\},
$$

and set $\mathcal{K}^{2}:=\mathcal{K} \times \mathcal{K}$

Definition 1.1. Given $\left(f_{0}, g_{0}\right) \in \mathcal{K}^{2}$, a pair $(f, g):[0, \infty) \rightarrow \mathcal{K}^{2}$ is a weak solution to $(1.1)$ if

(i) $(f, g) \in L_{\infty}\left(0, \infty ; L_{2}\left(\mathbb{R} ; \mathbb{R}^{2}\right)\right),(f, g) \in L_{2}\left(0, t ; H^{1}\left(\mathbb{R} ; \mathbb{R}^{2}\right)\right)$ for all $t>0$,

(ii) $(f, g) \in C\left([0, \infty) ; H^{-1}\left(\mathbb{R} ; \mathbb{R}^{2}\right)\right)$ with $(f, g)(0)=\left(f_{0}, g_{0}\right)$, 
and $(f, g)$ solves $(1.1 a)$ in the following sense:

$$
\left.\begin{array}{r}
\int_{\mathbb{R}} f(t) \xi \mathrm{d} x-\int_{\mathbb{R}} f_{0} \xi \mathrm{d} x+\int_{0}^{t} \int_{\mathbb{R}} f(\sigma)\left[(1+R) \partial_{x} f+R \partial_{x} g\right](\sigma) \partial_{x} \xi \mathrm{d} x \mathrm{~d} \sigma=0 \\
\int_{\mathbb{R}} g(t) \xi \mathrm{d} x-\int_{\mathbb{R}} g_{0} \xi \mathrm{d} x+R_{\mu} \int_{0}^{t} \int_{\mathbb{R}} g(\sigma)\left(\partial_{x} f+\partial_{x} g\right)(\sigma) \partial_{x} \xi \mathrm{d} x \mathrm{~d} \sigma=0
\end{array}\right\}
$$

for all $\xi \in C_{0}^{\infty}(\mathbb{R})$ and $t \geqslant 0$.

The existence of weak solutions to (1.1) is shown in [10] by a variational scheme. The proof relies on the observation that system $(1.1 a)$ is a gradient flow with respect to the 2-Wasserstein metric of the energy functional

$$
\mathcal{E}(f, g):=\frac{1}{2} \int_{\mathbb{R}}\left[f^{2}+R(f+g)^{2}\right] \mathrm{d} x .
$$

This approach actually extends to the two-dimensional setting as well as to a related fourth-order degenerate system that is also a thin-film approximation of the Muskat problem which additionally incorporates surface tension effects [11]. Note that the uniqueness of solutions to (1.1) is an open problem.

The main results of this paper are the following.

THEOREM 1.2 (finite speed of propagation). Let $(f, g)$ be a weak solution of (1.1). If $(f, g)$ satisfies the local energy estimate

$$
\begin{aligned}
\int_{\mathbb{R}}\left[f^{2}(T)\right. & \left.+R(f+g)^{2}(T)\right] \zeta^{2} \mathrm{~d} x \\
& \quad+\int_{0}^{T} \int_{\mathbb{R}}\left(f\left|(1+R) \partial_{x} f+R \partial_{x} g\right|^{2}+R R_{\mu} g\left|\partial_{x} f+\partial_{x} g\right|^{2}\right) \zeta^{2} \mathrm{~d} x \mathrm{~d} t \\
\leqslant & \int_{\mathbb{R}}\left[f^{2}(0)+R(f+g)^{2}(0)\right] \zeta^{2} \mathrm{~d} x \\
& +4 \int_{0}^{T} \int_{\mathbb{R}}\left[f((1+R) f+R g)^{2}+R R_{\mu} g(f+g)^{2}\right]\left|\partial_{x} \zeta\right|^{2} \mathrm{~d} x \mathrm{~d} t
\end{aligned}
$$

for all $\zeta \in W_{4}^{1}(\mathbb{R})$ as well as for $\zeta \equiv 1$, then $(f, g)$ has a finite speed of propagation. More precisely, if $a \geqslant 0, r_{0}>0$, and $\operatorname{supp}\left(f_{0}+g_{0}\right) \cap\left(a-r_{0}, a+r_{0}\right)=\emptyset$, then there exists a positive constant $C_{*}=C_{*}\left(R, R_{\mu}\right)$ such that

$$
\operatorname{supp}(f(T)+g(T)) \cap\left(a-\frac{1}{2} r_{0}, a+\frac{1}{2} r_{0}\right)=\emptyset \quad \text { for all } T \in\left(0, C_{*} r_{0}^{5 / 2} / \mathcal{E}^{1 / 2}\left(f_{0}, g_{0}\right)\right] .
$$

In particular, if $\operatorname{supp}\left(f_{0}+g_{0}\right) \subset\left[-b_{0}, b_{0}\right]$, with $b_{0}>0$, then there exists a positive constant $C^{*}=C^{*}\left(R_{\mu}, R, f_{0}, g_{0}\right)$ such that

$$
\operatorname{supp}(f(T)+g(T)) \subset\left[-b_{0}-C^{*} T^{1 / 3}, b_{0}+C^{*} T^{1 / 3}\right] \quad \text { for all } T>0 .
$$

We note that theorem 1.2 is only valid for weak solutions that also satisfy the local energy estimate (1.5). Unfortunately, we are as yet unable to derive it for arbitrary weak solutions, and it is unclear whether it holds in particular for the weak solutions constructed in [10]. We shall show in $\S 3$ that for each initial condition 
there is at least one weak solution to (1.1) satisfying the local estimate (1.5). To this end we shall adapt an approximation scheme from [6] that allows us to obtain a weak solution as a limit of classical solutions to a regularized version of the original system.

Note also that theorem 1.2 gives no clue regarding the finite speed of propagation for each component when taken separately.

\section{REMARK 1.3.}

(a) It is shown in [12] that system $(1.1 a)$ has self-similar solutions of the type

$$
\left[(t, x) \mapsto(1+t)^{-1 / 3}(F, G)\left((1+t)^{-1 / 3} x\right)\right] \text { for } t \geqslant 0 \text { and } x \in \mathbb{R},
$$

with compactly supported profiles $(F, G) \in H^{1}\left(\mathbb{R}, \mathbb{R}^{2}\right) \cap \mathcal{K}^{2}$. Hence, the estimate on the growth rate of the support obtained in theorem 1.2 matches that of the self-similar solutions and is likely to be optimal.

(b) The constant $C^{*}$ in the last statement of theorem 1.2 depends only on $f_{0}$ and $g_{0}$ through the energy $\mathcal{E}\left(f_{0}, g_{0}\right)$ and the second moments of $f_{0}$ and $g_{0}$.

Due to [4], a direct consequence of the local energy estimate (1.5) is the occurrence of waiting time phenomena.

THeOREM 1.4 (waiting time phenomena). Let $(f, g)$ be a weak solution of (1.1) such that (1.5) holds for all $\zeta \in W_{4}^{1}(\mathbb{R})$. Let $x_{0} \in \mathbb{R} \backslash \operatorname{supp}\left(f_{0}+g_{0}\right)$ be such that

$$
\limsup _{r \rightarrow 0} \frac{1}{r^{5}} \int_{x_{0}-r}^{x_{0}+r}\left[f_{0}^{2}+R\left(f_{0}+g_{0}\right)^{2}\right] \mathrm{d} x<\infty .
$$

Then there exists a positive time $T_{*}$ such that $x_{0} \in \overline{\mathbb{R} \backslash \operatorname{supp}(f(T)+g(T))}$ for all $T \in\left(0, T_{*}\right)$.

Let us now describe the content of this paper. Section 2 is devoted to the proof of the main results. While theorem 1.4 is a straightforward consequence of (1.5) and [4, theorem 1.2], the proof of theorem 1.2 requires several steps and is inspired by [3], which deals with the thin-film equation. We note that fewer estimates are available for (1.1) than for the equation studied in [3]. The last section is devoted to the existence of weak solutions to (1.1) that satisfy the local energy estimate (1.5).

\section{Finite speed of propagation}

Throughout this section, $(f, g)$ is a weak solution of (1.1) that satisfies the local energy estimate (1.5) and

$$
w:=\left[f^{2}+R(f+g)^{2}\right]^{3 / 4} .
$$

The function $w$ inherits some regularity properties of $(f, g)$ as shown in the following result.

Lemma 2.1. Given non-negative functions $u, v \in H^{1}(\mathbb{R})$, let

$$
z:=\left(u^{2}+R v^{2}\right)^{3 / 4} \text {. }
$$


Then $z \in H^{1}(\mathbb{R})$ and

$$
\partial_{x} z=\frac{3}{2} \frac{u \partial_{x} u+R v \partial_{x} v}{\mathbf{1}_{\{0\}}(z)+\left(u^{2}+R v^{2}\right)^{1 / 4}},
$$

where $\mathbf{1}_{E}$ is the characteristic function of the set $E$.

Proof. We choose positive functions $u_{n}, v_{n} \in C^{\infty}(\mathbb{R}) \cap H^{1}(\mathbb{R}), n \geqslant 1$, such that $u_{n} \rightarrow u$ and $v_{n} \rightarrow v$ in $H^{1}(\mathbb{R})$ and set

$$
z_{n}:=\left(u_{n}^{2}+R v_{n}^{2}\right)^{3 / 4}
$$

Obviously, $z_{n}^{4 / 3} \rightarrow z^{4 / 3}$ in $L_{1}(\mathbb{R})$ and it follows from the Hölder continuity of the function $\left[x \mapsto|x|^{3 / 4}\right]$ that

$$
\left|z_{n}-z\right|^{4 / 3} \leqslant\left|z_{n}^{4 / 3}-z^{4 / 3}\right| \text { for all } n \in \mathbb{N} .
$$

Hence, $z_{n} \rightarrow z$ in $L_{4 / 3}(\mathbb{R})$. We next note that the sequence $\left(z_{n}\right)_{n}$ is bounded in $H^{1}(\mathbb{R})$ so that it has a subsequence which converges weakly in $H^{1}(\mathbb{R})$ towards a limit coinciding with $z$ almost everywhere. Consequently, $z$ belongs to $H^{1}(\mathbb{R})$ and the formula for $\partial_{x} z$ follows by standard arguments.

We now derive from (1.5) a local energy estimate for the function $w$, defined in (2.1), which is at the heart of our analysis.

LEMMA 2.2. The function $w$ defined in (2.1) satisfies

$$
\begin{aligned}
\int_{\mathbb{R}} w^{4 / 3}(T) \zeta^{2} \mathrm{~d} x+C_{1} \int_{0}^{T} \int_{\mathbb{R}}\left|\partial_{x} w\right|^{2} \zeta^{2} \mathrm{~d} x \mathrm{~d} t & \\
& \leqslant \int_{\mathbb{R}} w^{4 / 3}(0) \zeta^{2} \mathrm{~d} x+C_{2} \int_{0}^{T} \int_{\mathbb{R}} w^{2}\left|\partial_{x} \zeta\right|^{2} \mathrm{~d} x \mathrm{~d} t
\end{aligned}
$$

for all $T>0$ and all $\zeta \in W_{4}^{1}(\mathbb{R})$. The constants $C_{1}$ and $C_{2}$ depend only on $R$ and $R_{\mu}$.

Proof. By lemma 2.1 the function $w$ belongs to $H^{1}(\mathbb{R})$ and

$$
\begin{aligned}
\left|\partial_{x} w\right|^{2}= & \left|\frac{f\left[(1+R) \partial_{x} f+R \partial_{x} g\right]+R g\left(\partial_{x} f+\partial_{x} g\right)}{\mathbf{1}_{\{0\}}(w)+\left(f^{2}+R(f+g)^{2}\right)^{1 / 4}}\right|^{2} \\
\leqslant & \frac{2 f}{\mathbf{1}_{\{0\}}(w)+\left(f^{2}+R(f+g)^{2}\right)^{1 / 2}} f\left|(1+R) \partial_{x} f+R \partial_{x} g\right|^{2} \\
& \quad+\frac{2 R^{2} g}{\mathbf{1}_{\{0\}}(w)+\left(f^{2}+R(f+g)^{2}\right)^{1 / 2}} g\left|\partial_{x} f+\partial_{x} g\right|^{2} \\
\leqslant & 2 \max \left\{1, \frac{\sqrt{R}}{R_{\mu}}\right\}\left[f\left|(1+R) \partial_{x} f+R \partial_{x} g\right|^{2}+R R_{\mu} g\left|\partial_{x} f+\partial_{x} g\right|^{2}\right] .
\end{aligned}
$$

In addition, since $w^{4 / 3} \geqslant \max \left\{(1+R) f^{2}, R g^{2}\right\}$, it holds that

$$
f((1+R) f+R g)^{2}+R R_{\mu} g(f+g)^{2} \leqslant 2 \max \left\{\frac{R_{\mu}}{\sqrt{R}}, \sqrt{1+R}\right\} w^{2} .
$$

Combining these two inequalities with (1.5) gives the claim. 
We next recall that the Gagliardo-Nirenberg inequality [14, theorem 1] states that there exists a constant $C>0$ such that

$$
\|v\|_{2} \leqslant C\left\|\partial_{x} v\right\|_{2}^{1 / 5}\|v\|_{4 / 3}^{4 / 5}+C\|v\|_{4 / 3} \quad \text { for all } v \in H^{1}((-1,1)) .
$$

Using a scaling argument, we deduce from the inequality above that, for $r>0$,

$$
\|v\|_{2} \leqslant C\left\|\partial_{x} v\right\|_{2}^{1 / 5}\|v\|_{4 / 3}^{4 / 5}+C r^{-1 / 4}\|v\|_{4 / 3} \quad \text { for all } v \in H^{1}((-r, r)) .
$$

A consequence of the Gagliardo-Nirenberg inequality (2.4) is the following interpolation inequality in the spirit of [3, lemma 10.1].

Lemma 2.3. There exists a $C_{3}>0$ such that, given $r>0$ and $v \in H^{1}((-r, r))$,

$$
\|v\|_{2}^{2} \leqslant C_{3}\left\|\partial_{x} v\right\|_{2}^{14 / 11} I_{r}^{6 / 11}+C_{3} r^{-7 / 2} I_{r}^{3 / 2}
$$

holds, where

$$
I_{r}:=\int_{-r}^{r}(r-|x|)_{+}^{2}|v(x)|^{4 / 3} \mathrm{~d} x .
$$

Proof. We pick $\rho \in(0, r)$ arbitrarily and infer from the Hölder inequality that

$$
\begin{aligned}
\int_{-r}^{r}|v|^{4 / 3} \mathrm{~d} x & =\int_{-\rho}^{\rho}|v|^{4 / 3} \mathrm{~d} x+\int_{\{\rho<|x|<r\}}|v|^{4 / 3} \mathrm{~d} x \\
& \leqslant \frac{1}{(r-\rho)^{2}} \int_{-\rho}^{\rho}(r-|x|)_{+}^{2}|v|^{4 / 3} \mathrm{~d} x+2(r-\rho)^{1 / 3}\|v\|_{2}^{4 / 3} .
\end{aligned}
$$

We now choose $\rho \in(0, r)$ such that

$$
\frac{1}{(r-\rho)^{2}} \int_{-\rho}^{\rho}(r-|x|)_{+}^{2}|v|^{4 / 3} \mathrm{~d} x=2(r-\rho)^{1 / 3}\|v\|_{2}^{4 / 3},
$$

and obtain

$$
\|v\|_{4 / 3} \leqslant 4\|v\|_{2}^{6 / 7} I_{r}^{3 / 28}
$$

Using (2.4) and (2.6) yields

$$
\|v\|_{2}^{2} \leqslant C\left\|\partial_{x} v\right\|_{2}^{2 / 5}\left(\|v\|_{2}^{2}\right)^{24 / 35} I_{r}^{6 / 35}+C r^{-1 / 2}\left(\|v\|_{2}^{2}\right)^{6 / 7} I_{r}^{3 / 14}
$$

and thus

$$
\left(\|v\|_{2}^{2}\right)^{11 / 35} \leqslant C\left\|\partial_{x} v\right\|_{2}^{2 / 5} I_{r}^{6 / 35}+C r^{-1 / 2}\left(\|v\|_{2}^{2}\right)^{6 / 35} I_{r}^{3 / 14} .
$$

By Young's inequality we get

$$
\begin{aligned}
\|v\|_{2}^{2} & \leqslant C\left\|\partial_{x} v\right\|_{2}^{14 / 11} I_{r}^{6 / 11}+C r^{-35 / 22}\left(\|v\|_{2}^{2}\right)^{6 / 11} I_{r}^{15 / 22} \\
& \leqslant C\left\|\partial_{x} v\right\|_{2}^{14 / 11} I_{r}^{6 / 11}+\frac{1}{2}\|v\|_{2}^{2}+C r^{-7 / 2} I_{r}^{3 / 2}
\end{aligned}
$$

and the proof is complete. 
We now introduce additional notation. For $r>0$ and $T>0$ we set

$$
\begin{aligned}
u_{k}(r, T) & :=\int_{0}^{T} \int_{-r}^{r}\left|\partial_{x} w(t, x)\right|^{2}(r-|x|)_{+}^{k} \mathrm{~d} x \mathrm{~d} t \quad \text { for } k \in\{0,1,2\} \\
I(r, T) & :=\sup _{t \in(0, T)} \int_{-r}^{r} w^{4 / 3}(t, x)(r-|x|)_{+}^{2} \mathrm{~d} x
\end{aligned}
$$

where $w$ is defined in (2.1). We first derive from (2.2) an inequality relating $I(r, T)$, $u_{0}(r, T)$ and $u_{2}(r, T)$ under suitable constraints on $r$ and $T$.

Lemma 2.4. Consider $r_{0}>0$ such that $\operatorname{supp}\left(f_{0}+g_{0}\right) \cap\left(-r_{0}, r_{0}\right)=\emptyset$. There are positive constants $C_{4}$ and $C_{5}$ such that, if $T_{0}>0$ is such that

$$
C_{4} T_{0}\left(\frac{1}{2} r_{0}\right)^{-7 / 2} I^{1 / 2}\left(r_{0}, T_{0}\right) \leqslant \frac{1}{2}
$$

then

$$
\frac{1}{3} I(r, T)+u_{2}(r, T) \leqslant C_{5} T^{4 / 5} u_{0}^{7 / 5}(r, T)
$$

for all $\frac{1}{2} r_{0} \leqslant r \leqslant r_{0}$ and $0<T \leqslant T_{0}$.

Proof. Let $T \in\left(0, T_{0}\right]$ and $r \in\left(0, r_{0}\right]$. Setting $\zeta(x):=(r-|x|)_{+}, x \in \mathbb{R}$, we observe that the assumptions on $f_{0}+g_{0}$ guarantee that $\zeta^{2}(x) w^{4 / 3}(0, x)=0$ for $x \in \mathbb{R}$, and we infer from $(2.2)$ that

$$
\int_{\mathbb{R}} w^{4 / 3}(t) \zeta^{2} \mathrm{~d} x+C_{1} \int_{0}^{t} \int_{\mathbb{R}}\left|\partial_{x} w\right|^{2} \zeta^{2} \mathrm{~d} x \mathrm{~d} s \leqslant C_{2} \int_{0}^{t} \int_{-r}^{r} w^{2} \mathrm{~d} x \mathrm{~d} s
$$

for all $t \in(0, T)$. Hence, there exists a constant $C>0$ such that

$$
I(r, T)+u_{2}(r, T) \leqslant C \int_{0}^{T} \int_{-r}^{r} w^{2} \mathrm{~d} x \mathrm{~d} s .
$$

Using (2.5) and the Hölder inequality, it follows that

$$
\begin{aligned}
I(r, T)+u_{2}(r, T) & \leqslant C T r^{-7 / 2} I^{3 / 2}(r, T)+C I^{6 / 11}(r, T) \int_{0}^{T}\left\|\partial_{x} w(s)\right\|_{L_{2}((-r, r))}^{14 / 11} \mathrm{~d} s \\
& \leqslant C_{4} \operatorname{Tr}^{-7 / 2} I^{3 / 2}(r, T)+C T^{4 / 11} I^{6 / 11}(r, T) u_{0}^{7 / 11}(r, T) .
\end{aligned}
$$

Since $I(r, T)$ is a non-decreasing function in both variables $r$ and $T$, the property (2.7), together with Young's inequality and the above inequality, leads us to

$$
I(r, T)+u_{2}(r, T) \leqslant \frac{1}{2} I(r, T)+\frac{1}{6} I(r, T)+C_{5} T^{4 / 5} u_{0}^{7 / 5}(r, T)
$$

for all $\frac{1}{2} r_{0} \leqslant r \leqslant r_{0}$ and $0<T \leqslant T_{0}$. This completes the proof.

We are now in a position to prove our main results.

Proof of theorem 1.2. Since (1.1) is invariant with respect to translation, we may assume that $a=0$, so that $\operatorname{supp}\left(f_{0}+g_{0}\right) \cap\left(-r_{0}, r_{0}\right)=\emptyset$. Then $w^{4 / 3}(0, x)=0$ for $x \in\left(-r_{0}, r_{0}\right)$ and $I\left(r_{0}, t\right) \rightarrow 0$ as $t \rightarrow 0$ by (2.2). Consequently, there exists a $T_{0}>0$ such that the condition (2.7) in lemma 2.4 is satisfied. 
Let $T \in\left(0, T_{0}\right)$. In view of

$$
\left[x \mapsto\left\|\partial_{x} w(\cdot, x)\right\|_{L_{2}(0, T)}^{2}\right] \in L_{1}(\mathbb{R}),
$$

we have

$$
\partial_{r} u_{2}(r, T)=2 u_{1}(r, T), \quad \partial_{r} u_{1}(r, T)=u_{0}(r, T)
$$

for almost every (a.e.) $r \in\left(0, r_{0}\right)$, and the Hölder inequality yields

$$
u_{1}(r, T) \leqslant u_{2}^{1 / 2}(r, T) u_{0}^{1 / 2}(r, T) .
$$

This inequality, together with (2.8), which is valid here due to the choice of $T_{0}$, gives

$$
u_{1}(r, T) \leqslant C T^{2 / 5} u_{0}^{6 / 5}(r, T)=C T^{2 / 5}\left(\partial_{r} u_{1}(r, T)\right)^{6 / 5} \quad \text { for a.e. } r \in\left(\frac{1}{2} r_{0}, r_{0}\right) .
$$

Equivalently,

$$
u_{1}^{5 / 6}(r, T) \leqslant \frac{1}{6 C_{6}} T^{1 / 3} \partial_{r} u_{1}(r, T) \quad \text { for a.e. } r \in\left(\frac{1}{2} r_{0}, r_{0}\right) .
$$

Taking a smaller value of $T_{0}$ if necessary, we further assume that

$$
T_{0}^{1 / 3} u_{1}^{1 / 6}\left(r_{0}, T_{0}\right) \leqslant \frac{1}{2} C_{6} r_{0} .
$$

Let $T \in\left(0, T_{0}\right]$ and assume for the following argument by contradiction that $u_{1}\left(\frac{1}{2} r_{0}, T\right)>0$. Together with the monotonicity properties of $u_{1}$ this implies that $u_{1}(r, T)>0$ for all $r \in\left[\frac{1}{2} r_{0}, r_{0}\right]$. Due to this positivity property, we infer from (2.10) that

$$
C_{6} T^{-1 / 3} \leqslant \partial_{r}\left(u_{1}^{1 / 6}\right)(r, T) \text { for a.e. } r \in\left(\frac{1}{2} r_{0}, r_{0}\right) .
$$

After integration we end up with

$$
C_{6} T^{-1 / 3}\left(r_{0}-r\right) \leqslant u_{1}^{1 / 6}\left(r_{0}, T\right)-u_{1}^{1 / 6}(r, T),
$$

or, equivalently,

$$
T^{1 / 3} u_{1}^{1 / 6}(r, T) \leqslant T^{1 / 3} u_{1}^{1 / 6}\left(r_{0}, T\right)-C_{6}\left(r_{0}-r\right), \quad r \in\left[\frac{1}{2} r_{0}, r_{0}\right] .
$$

Taking $r=\frac{1}{2} r_{0}$ in $(2.12)$ gives

$$
0<T_{0}^{1 / 3} u_{1}^{1 / 6}\left(r_{0}, T_{0}\right)-\frac{1}{2} C_{6} r_{0},
$$

and contradicts (2.11). Therefore, $u_{1}\left(\frac{1}{2} r_{0}, T\right)=0$ and it follows from (2.9) that $u_{0}\left(\frac{1}{2} r_{0}, T\right)=0$ for all $T \in\left(0, T_{0}\right]$. Recalling $(2.8)$, we find that $I\left(\frac{1}{2} r_{0}, T\right)=0$ for all $T \in\left(0, T_{0}\right]$.

We further note that, in view of theorem 3.1(b) and (2.3),

$$
\begin{gathered}
u_{1}(r, T) \leqslant r \int_{0}^{T}\left\|\partial_{x} w(t)\right\|_{2}^{2} \mathrm{~d} t \leqslant C r\left[\mathcal{E}\left(f_{0}, g_{0}\right)-\mathcal{E}(f(T), g(T))\right] \leqslant C r \mathcal{E}\left(f_{0}, g_{0}\right), \\
I(r, T) \leqslant r^{2} \sup _{t \in[0, T]}\|w(t)\|_{4 / 3}^{4 / 3} \leqslant r^{2} \sup _{t \in[0, T]} \mathcal{E}(f(t), g(t)) \leqslant r^{2} \mathcal{E}\left(f_{0}, g_{0}\right),
\end{gathered}
$$


so that (2.7) and (2.11) are satisfied provided that $T_{0}=C_{*} r_{0}^{5 / 2} / \mathcal{E}^{1 / 2}\left(f_{0}, g_{0}\right)$ for a sufficiently small constant $C_{*}>0$ depending only on $R$ and $R_{\mu}$. This proves the first claim of theorem 1.2.

Finally, let $\operatorname{supp}\left(f_{0}+g_{0}\right) \subset\left[-b_{0}, b_{0}\right]$, with $b_{0}>0$, and let $T>0$ be fixed. Choosing $r_{0}=\left(T \mathcal{E}^{1 / 2}\left(f_{0}, g_{0}\right) / C_{*}\right)^{2 / 5}$, we have that, for each $a \geqslant b_{0}+\left(T \mathcal{E}^{1 / 2}\left(f_{0}, g_{0}\right) / C_{*}\right)^{2 / 5}$ or $a \leqslant-b_{0}-\left(T \mathcal{E}^{1 / 2}\left(f_{0}, g_{0}\right) / C_{*}\right)^{2 / 5}$,

$$
\operatorname{supp}\left(f_{0}+g_{0}\right) \cap\left(a-r_{0}, a+r_{0}\right)=\emptyset .
$$

We then infer from the first statement of theorem 1.2 that $\operatorname{supp}(f(T)+g(T)) \cap$ $\left(a-\frac{1}{2} r_{0}, a+\frac{1}{2} r_{0}\right)=\emptyset$, from which it follows that

$$
\begin{array}{r}
\operatorname{supp}(f(T)+g(T)) \subset\left[-b_{0}-\frac{T^{2 / 5} \mathcal{E}^{1 / 5}\left(f_{0}, g_{0}\right)}{2 C_{*}^{2 / 5}}, b_{0}+\frac{T^{2 / 5} \mathcal{E}^{1 / 5}\left(f_{0}, g_{0}\right)}{2 C_{*}^{2 / 5}}\right] \\
\quad \text { for all } T>0 .
\end{array}
$$

Consequently, $(f(T)+g(T))$ is compactly supported for each $T \geqslant 0$ and we set

$$
\beta(T):=\max \left\{b_{0}, \sup (\operatorname{supp}(f(T)+g(T)))\right\} .
$$

It then follows that $\beta(T) \rightarrow \beta(0)=b_{0}$ as $T \rightarrow 0$. Since problem (1.1 $\left.a\right)$ is autonomous, the estimate (2.13) yields

$$
\beta\left(T_{2}\right)-\beta\left(T_{1}\right) \leqslant \frac{\left(T_{2}-T_{1}\right)^{2 / 5} \mathcal{E}^{1 / 5}\left(f\left(T_{1}\right), g\left(T_{1}\right)\right)}{2 C_{*}^{2 / 5}} \text { for all } T_{2}>T_{1} .
$$

Besides, we know from [12, theorem 4.1(iv)] (after rescaling) that

$$
\begin{aligned}
\mathcal{E}(f(t), g(t)) & \leqslant(1+t)^{-1 / 3}\left[\mathcal{E}\left(f_{0}, g_{0}\right)+\frac{1}{6} \int_{\mathbb{R}}\left(f_{0}+\frac{R}{R_{\mu}} g_{0}\right) x^{2} \mathrm{~d} x\right] \\
& \leqslant C t^{-1 / 3} \quad \text { for all } t>0 .
\end{aligned}
$$

Combining (2.14) and (2.15) yields

$$
\beta\left(T_{2}\right)-\beta\left(T_{1}\right) \leqslant C\left(T_{2}-T_{1}\right)^{2 / 5} T_{1}^{-1 / 15} \text { for all } T_{2}>T_{1}>0 .
$$

We are now in the position to apply [3, lemma 7.6] to the above functional inequality and conclude that there exists a positive constant $C^{*}$ depending only on $R, R_{\mu}, f_{0}$, and $g_{0}$ such that

$$
\beta(T) \leqslant b_{0}+C^{*} T^{1 / 3} \quad \text { for all } T>0,
$$

which is the expected propagation rate. The estimate for the expansion of the left boundary of the support is derived in a similar way.

Proof of theorem 1.4. Invoking (2.2), theorem 1.4 is a particular case of the more general result [4, theorem 1.2], which we apply with $k=1, p=2$ and $q=\frac{4}{3}$.

\section{Weak solutions satisfying the local energy estimate}

As mentioned in $\S 1$, we now check that there exists at least a weak solution to (1.1) satisfying the local energy estimate (1.5). 
Theorem 3.1 (existence of weak solutions). Given $\left(f_{0}, g_{0}\right) \in \mathcal{K}^{2}$, where $\mathcal{K}^{2}$ is defined in (1.2), there exists at least a weak solution $(f, g)$ to (1.1), satisfying the local energy estimate (1.5) as well as the following estimates:

(a) $\|f(T)\|_{1}=\left\|f_{0}\right\|_{1},\|g(T)\|_{1}=\left\|g_{0}\right\|_{1}$,

(b) $\mathcal{H}(f(T), g(T))+\frac{R}{1+2 R} \int_{0}^{T} \int_{\mathbb{R}}\left[\left|\partial_{x} f\right|^{2}+R\left|\partial_{x}(f+g)\right|^{2}\right] \mathrm{d} x \mathrm{~d} t \leqslant \mathcal{H}\left(f_{0}, g_{0}\right)$,

(c) $\mathcal{E}(f(T), g(T))+\frac{1}{2} \int_{0}^{T} \int_{\mathbb{R}}\left[f\left((1+R) \partial_{x} f+R \partial_{x} g\right)^{2}+R R_{\mu} g\left(\partial_{x} f+\partial_{x} g\right)^{2}\right] \mathrm{d} x \mathrm{~d} t$ $\leqslant \mathcal{E}\left(f_{0}, g_{0}\right)$

for all $T \in(0, \infty)$. The energy functional $\mathcal{E}$ is given by (1.4) and the entropy functional $\mathcal{H}$ is defined as

$$
\mathcal{H}(f, g):=\int_{\mathbb{R}}\left(f \ln f+\frac{R}{R_{\mu}} g \ln g\right) \mathrm{d} x .
$$

The remainder of this section is devoted to the proof of theorem 3.1. We split the proof of theorem 3.1 into two steps: we first truncate the spatial domain to a finite interval $(-L, L)$, for some arbitrary $L>0$, and then introduce a regularized system having global classical solutions.

\subsection{A regularized problem}

To be more precise, given $L>0$ and $\varepsilon \in(0,1)$, we define the Hilbert space

$$
H_{\mathcal{B}}^{2}:=\left\{u \in H^{2}((-L, L)): \partial_{x} u( \pm L)=0\right\}
$$

and we note that the elliptic operator $\left(1-\varepsilon^{2} \partial_{x}^{2}\right): H_{\mathcal{B}}^{2} \rightarrow L_{2}((-L, L))$ is an isomorphism. Setting

$$
\mathcal{R}_{\varepsilon}[u]:=\left(1-\varepsilon^{2} \partial_{x}^{2}\right)^{-1} u \in H_{\mathcal{B}}^{2} \quad \text { for } u \in L_{2}((-L, L)),
$$

we consider the following regularized problem:

$$
\left.\begin{array}{l}
\partial_{t} f_{\varepsilon}=(1+R) \partial_{x}\left(f_{\varepsilon} \partial_{x} f_{\varepsilon}\right)+R \partial_{x}\left(\left(f_{\varepsilon}-\varepsilon\right) \partial_{x} \mathcal{R}_{\varepsilon}\left[g_{\varepsilon}\right]\right), \\
\partial_{t} g_{\varepsilon}=R_{\mu} \partial_{x}\left(\left(g_{\varepsilon}-\varepsilon\right) \partial_{x} \mathcal{R}_{\varepsilon}\left[f_{\varepsilon}\right]\right)+R_{\mu} \partial_{x}\left(g_{\varepsilon} \partial_{x} g_{\varepsilon}\right)
\end{array}\right\}
$$

for $(t, x) \in(0, \infty) \times(-L, L)$, supplemented with homogeneous Neumann boundary conditions

$$
\partial_{x} f_{\varepsilon}(t, \pm L)=\partial_{x} g_{\varepsilon}(t, \pm L)=0, \quad t \in(0, \infty),
$$

and with regularized initial data

$$
f_{\varepsilon}(0)=f_{0 \varepsilon}:=\mathcal{R}_{\varepsilon}\left[f_{0} \mathbf{1}_{(-L, L)}\right]+\varepsilon, \quad g_{\varepsilon}(0)=g_{0 \varepsilon}:=\mathcal{R}_{\varepsilon}\left[g_{0} \mathbf{1}_{(-L, L)}\right]+\varepsilon .
$$

Clearly, the regularized initial data satisfy $\left(f_{0 \varepsilon}, g_{0 \varepsilon}\right) \in H_{\mathcal{B}}^{2} \times H_{\mathcal{B}}^{2}$ and

$$
f_{0 \varepsilon} \geqslant \varepsilon, \quad g_{0 \varepsilon} \geqslant \varepsilon .
$$

The solvability of problem (3.2) is studied in [6, theorem 2.1] with the help of the quasilinear parabolic theory developed in [1] and we recall the result now. 
Proposition 3.2. The problem (3.2) has a unique non-negative classical solution

$$
f_{\varepsilon}, g_{\varepsilon} \in C\left([0, \infty) ; H^{1}((-L, L))\right) \cap C\left((0, \infty) ; H_{\mathcal{B}}^{2}\right) \cap C^{1}\left((0, \infty) ; L_{2}((-L, L))\right) .
$$

Moreover, we have

$$
f_{\varepsilon} \geqslant \varepsilon, \quad g_{\varepsilon} \geqslant \varepsilon \quad \text { for all }(t, x) \in(0, \infty) \times(-L, L),
$$

and

$$
\begin{aligned}
\left\|f_{\varepsilon}(t)\right\|_{1} & =\left\|f_{0 \varepsilon}\right\|_{1}=\left\|f_{0} \mathbf{1}_{(-L, L)}\right\|_{1}+2 \varepsilon L, \\
\left\|g_{\varepsilon}(t)\right\|_{1} & =\left\|g_{0 \varepsilon}\right\|_{1}=\left\|g_{0} \mathbf{1}_{(-L, L)}\right\|_{1}+2 \varepsilon L
\end{aligned}
$$

for all $t \geqslant 0$.

The solutions constructed in proposition 3.2 enjoy additional properties (see $[6$, lemmas 2.4 and 2.6]).

LEMma 3.3. Given $T \in(0, \infty)$, the following hold:

$$
\begin{aligned}
\mathcal{H}\left(f_{\varepsilon}(T), g_{\varepsilon}(T)\right)+\int_{0}^{T} \int_{-L}^{L}\left(\frac{\left|\partial_{x} f_{\varepsilon}\right|^{2}}{2}+\frac{R}{1+2 R}\left|\partial_{x} g_{\varepsilon}\right|^{2}\right) \mathrm{d} x \mathrm{~d} t & \\
& \leqslant \mathcal{H}\left(f_{\varepsilon}(0), g_{\varepsilon}(0)\right)
\end{aligned}
$$

and

$$
\begin{array}{r}
\mathcal{E}_{\varepsilon}\left(f_{\varepsilon}(T), g_{\varepsilon}(T)\right)+\int_{0}^{T} \int_{-L}^{L}\left[f_{\varepsilon}\left|(1+R) \partial_{x} f_{\varepsilon}+R \partial_{x} G_{\varepsilon}\right|^{2}+R R_{\mu} g_{\varepsilon}\left|\partial_{x}\left(F_{\varepsilon}+g_{\varepsilon}\right)\right|^{2}\right] \mathrm{d} x \mathrm{~d} t \\
\leqslant \mathcal{E}_{\varepsilon}\left(f_{0 \varepsilon}, g_{0 \varepsilon}\right)+\varepsilon C_{2} \int_{0}^{T} \int_{-L}^{L}\left(\left|\partial_{x} f_{\varepsilon}\right|^{2}+\left|\partial_{x} g_{\varepsilon}\right|^{2}\right) \mathrm{d} x \mathrm{~d} t, \quad(3.5)
\end{array}
$$

with $F_{\varepsilon}:=\mathcal{R}_{\varepsilon}\left[f_{\varepsilon}\right], G_{\varepsilon}:=\mathcal{R}_{\varepsilon}\left[g_{\varepsilon}\right]$

$$
\begin{aligned}
\mathcal{E}_{\varepsilon}\left(f_{\varepsilon}, g_{\varepsilon}\right) & :=\frac{1}{2}\left[(1+R)\left\|f_{\varepsilon}\right\|_{2}^{2}+R\left\|g_{\varepsilon}\right\|_{2}^{2}+R \int_{-L}^{L}\left(F_{\varepsilon} g_{\varepsilon}+G_{\varepsilon} f_{\varepsilon}\right) \mathrm{d} x\right], \\
\mathcal{H}\left(f_{\varepsilon}, g_{\varepsilon}\right) & :=\int_{-L}^{L}\left(f_{\varepsilon} \ln f_{\varepsilon}+\frac{R}{R_{\mu}} g_{\varepsilon} \ln g_{\varepsilon}\right) \mathrm{d} x .
\end{aligned}
$$

As a consequence of proposition 3.2 and lemma 3.3, the following result is proved in $[6]$.

Proposition 3.4 (weak solutions on a finite interval). There exist a sequence $\varepsilon_{k} \rightarrow 0$ and a pair $(f, g)$ satisfying

(i) $f \geqslant 0, g \geqslant 0$ in $(0, \infty) \times(-L, L)$,

(ii) $f, g \in L_{\infty}\left(0, \infty ; L_{2}((-L, L))\right) \cap L_{2}\left(0, T ; H^{1}((-L, L))\right)$ for all $T>0$,

(iii) $f_{\varepsilon_{k}} \rightarrow f, g_{\varepsilon_{k}} \rightarrow g$ in $L_{2}((0, T) \times(-L, L))$, 
and

$$
\begin{aligned}
& \int_{-L}^{L} f(T) \xi \mathrm{d} x-\int_{-L}^{L} f_{0} \xi \mathrm{d} x=-\int_{0}^{T} \int_{-L}^{L} f\left((1+R) \partial_{x} f+R \partial_{x} g\right) \partial_{x} \xi \mathrm{d} x \mathrm{~d} t \\
& \int_{-L}^{L} g(T) \xi \mathrm{d} x-\int_{-L}^{L} g_{0} \xi \mathrm{d} x=-R_{\mu} \int_{0}^{T} \int_{-L}^{L} g\left(\partial_{x} f+\partial_{x} g\right) \partial_{x} \xi \mathrm{d} x \mathrm{~d} t
\end{aligned}
$$

for all $\xi \in W_{4}^{1}((-L, L))$ and all $T>0$. Moreover,

(a) $\|f(T)\|_{1}=\left\|f_{0} \mathbf{1}_{(-L, L)}\right\|_{1},\|g(T)\|_{1}=\left\|g_{0} \mathbf{1}_{(-L, L)}\right\|_{1}$,

(b) $\mathcal{H}(f(T), g(T))+\int_{0}^{T} \int_{-L}^{L}\left[\frac{\left|\partial_{x} f\right|^{2}}{2}+\frac{R}{1+2 R}\left|\partial_{x} g\right|^{2}\right] \mathrm{d} x \mathrm{~d} t \leqslant \mathcal{H}\left(f_{0}, g_{0}\right)$,

(c) $\mathcal{E}(f(T), g(T))+\int_{0}^{T} \int_{-L}^{L}\left[f\left((1+R) \partial_{x} f+R \partial_{x} g\right)^{2}+R R_{\mu} g\left(\partial_{x} f+\partial_{x} g\right)^{2}\right] \mathrm{d} x \mathrm{~d} t$

$$
\leqslant \mathcal{E}\left(f_{0}, g_{0}\right)
$$

for all $T \in(0, \infty)$.

\subsection{A local energy estimate}

We now derive a local version of the inequality in proposition 3.4(c).

Lemma 3.5. Let $(f, g)$ be the limit of $\left(\left(f_{\varepsilon_{k}}, g_{\varepsilon_{k}}\right)\right)_{k}$ found in proposition 3.4. Then

$$
\begin{aligned}
& \int_{-L}^{L}\left[f^{2}(T)\right.\left.+R(f+g)^{2}(T)\right] \zeta^{2} \mathrm{~d} x \\
&+\int_{0}^{T} \int_{-L}^{L}\left(f\left|(1+R) \partial_{x} f+R \partial_{x} g\right|^{2}+R R_{\mu} g\left|\partial_{x} f+\partial_{x} g\right|^{2}\right) \zeta^{2} \mathrm{~d} x \mathrm{~d} t \\
& \leqslant \int_{-L}^{L}\left[f^{2}(0)+R(f+g)^{2}(0)\right] \zeta^{2} \mathrm{~d} x \\
& \quad+4 \int_{0}^{T} \int_{-L}^{L}\left[f((1+R) f+R g)^{2}+R R_{\mu} g(f+g)^{2}\right]\left|\partial_{x} \zeta\right|^{2} \mathrm{~d} x \mathrm{~d} t
\end{aligned}
$$

for all $T>0$ and all $\zeta \in W_{4}^{1}((-L, L))$.

Proof. We set

$$
U_{\varepsilon}:=\sqrt{f_{\varepsilon}} \partial_{x}\left[(1+R) f_{\varepsilon}+R G_{\varepsilon}\right], \quad V_{\varepsilon}:=\sqrt{g_{\varepsilon}} \partial_{x}\left[F_{\varepsilon}+g_{\varepsilon}\right],
$$

and prove first the claim (3.8) for $\zeta \in C_{0}^{\infty}((-L, L))$. We multiply the first equation of $(3.2 a)$ by $\left((1+R) f_{\varepsilon}+R G_{\varepsilon}\right) \zeta^{2}$ and integrate over $(-L, L)$ to obtain

$$
\begin{aligned}
\int_{-L}^{L} \partial_{t} f_{\varepsilon}\left((1+R) f_{\varepsilon}\right. & \left.+R G_{\varepsilon}\right) \zeta^{2} \mathrm{~d} x \\
& =-\int_{-L}^{L} \sqrt{f_{\varepsilon}} U_{\varepsilon} \partial_{x}\left[\left((1+R) f_{\varepsilon}+R G_{\varepsilon}\right) \zeta^{2}\right] \mathrm{d} x+I_{1, \varepsilon}
\end{aligned}
$$


with

$$
I_{1, \varepsilon}:=\varepsilon R \int_{-L}^{L} \partial_{x} G_{\varepsilon} \partial_{x}\left[\left((1+R) f_{\varepsilon}+R G_{\varepsilon}\right) \zeta^{2}\right] \mathrm{d} x .
$$

Similarly, multiplying the second equation of $(3.2 a)$ by $R\left(F_{\varepsilon}+g_{\varepsilon}\right) \zeta^{2}$ and integrating over $(-L, L)$ give

$$
R \int_{-L}^{L} \partial_{t} g_{\varepsilon}\left(F_{\varepsilon}+g_{\varepsilon}\right) \zeta^{2} \mathrm{~d} x=-R R_{\mu} \int_{-L}^{L} \sqrt{g_{\varepsilon}} V_{\varepsilon} \partial_{x}\left[\left(F_{\varepsilon}+g_{\varepsilon}\right) \zeta^{2}\right] \mathrm{d} x+I_{2, \varepsilon}
$$

with

$$
I_{2, \varepsilon}:=\varepsilon R R_{\mu} \int_{-L}^{L} \partial_{x} F_{\varepsilon} \partial_{x}\left[\left(F_{\varepsilon}+g_{\varepsilon}\right) \zeta^{2}\right] \mathrm{d} x .
$$

We now observe that

$$
\begin{aligned}
\int_{-L}^{L} \partial_{t} f_{\varepsilon}\left((1+R) f_{\varepsilon}+R G_{\varepsilon}\right) \zeta^{2} \mathrm{~d} x & +R \int_{-L}^{L} \partial_{t} g_{\varepsilon}\left(F_{\varepsilon}+g_{\varepsilon}\right) \zeta^{2} \mathrm{~d} x \\
= & \frac{1+R}{2} \frac{\mathrm{d}}{\mathrm{d} t}\left\|f_{\varepsilon} \zeta\right\|_{2}^{2}+\frac{R}{2} \frac{\mathrm{d}}{\mathrm{d} t}\left\|g_{\varepsilon} \zeta\right\|_{2}^{2}+R J_{\varepsilon}
\end{aligned}
$$

with

$$
\begin{aligned}
J_{\varepsilon}:= & \int_{-L}^{L}\left(G_{\varepsilon} \partial_{t} f_{\varepsilon}+F_{\varepsilon} \partial_{t} g_{\varepsilon}\right) \zeta^{2} \mathrm{~d} x \\
= & \frac{\mathrm{d}}{\mathrm{d} t} \int_{-L}^{L}\left(F_{\varepsilon} G_{\varepsilon}+\varepsilon^{2} \partial_{x} F_{\varepsilon} \partial_{x} G_{\varepsilon}\right) \zeta^{2} \mathrm{~d} x \\
& \quad+2 \varepsilon^{2} \int_{-L}^{L}\left(G_{\varepsilon} \partial_{x} \partial_{t} F_{\varepsilon}+F_{\varepsilon} \partial_{x} \partial_{t} G_{\varepsilon}\right) \zeta \partial_{x} \zeta \mathrm{d} x .
\end{aligned}
$$

Recalling that $\zeta \in C_{0}^{\infty}((-L, L))$, we have

$$
\begin{aligned}
\int_{-L}^{L}\left(F_{\varepsilon} G_{\varepsilon}+\varepsilon^{2} \partial_{x} F_{\varepsilon} \partial_{x} G_{\varepsilon}\right) \zeta^{2} \mathrm{~d} x & \\
= & \frac{1}{2} \int_{-L}^{L}\left(F_{\varepsilon} G_{\varepsilon}+\varepsilon^{2} \partial_{x} F_{\varepsilon} \partial_{x} G_{\varepsilon}\right) \zeta^{2} \mathrm{~d} x \\
& +\frac{1}{2} \int_{-L}^{L}\left(F_{\varepsilon} G_{\varepsilon}+\varepsilon^{2} \partial_{x} F_{\varepsilon} \partial_{x} G_{\varepsilon}\right) \zeta^{2} \mathrm{~d} x \\
= & \frac{1}{2} \int_{-L}^{L}\left(F_{\varepsilon} G_{\varepsilon}-\varepsilon^{2} G_{\varepsilon} \partial_{x}^{2} F_{\varepsilon}\right) \zeta^{2} \mathrm{~d} x \\
& +\frac{1}{2} \int_{-L}^{L}\left(F_{\varepsilon} G_{\varepsilon}-\varepsilon^{2} F_{\varepsilon} \partial_{x}^{2} G_{\varepsilon}\right) \zeta^{2} \mathrm{~d} x \\
& \quad-\varepsilon^{2} \int_{-L}^{L} G_{\varepsilon} \partial_{x} F_{\varepsilon} \zeta \partial_{x} \zeta \mathrm{d} x-\varepsilon^{2} \int_{-L}^{L} F_{\varepsilon} \partial_{x} G_{\varepsilon} \zeta \partial_{x} \zeta \mathrm{d} x \\
= & \frac{1}{2} \int_{-L}^{L}\left(F_{\varepsilon} g_{\varepsilon}+G_{\varepsilon} f_{\varepsilon}\right) \zeta^{2} \mathrm{~d} x+\varepsilon^{2} \int_{-L}^{L} F_{\varepsilon} G_{\varepsilon} \partial_{x}\left(\zeta \partial_{x} \zeta\right) \mathrm{d} x
\end{aligned}
$$


while

$$
\begin{aligned}
\int_{-L}^{L}\left(G_{\varepsilon} \partial_{x} \partial_{t} F_{\varepsilon}+F_{\varepsilon} \partial_{x} \partial_{t} G_{\varepsilon}\right) \zeta \partial_{x} \zeta \mathrm{d} x= & -\int_{-L}^{L}\left(\partial_{t} F_{\varepsilon} \partial_{x} G_{\varepsilon}+\partial_{x} F_{\varepsilon} \partial_{t} G_{\varepsilon}\right) \zeta \partial_{x} \zeta \mathrm{d} x \\
& -\int_{-L}^{L}\left(G_{\varepsilon} \partial_{t} F_{\varepsilon}+F_{\varepsilon} \partial_{t} G_{\varepsilon}\right) \partial_{x}\left(\zeta \partial_{x} \zeta\right) \mathrm{d} x \\
= & -\int_{-L}^{L}\left(\partial_{t} F_{\varepsilon} \partial_{x} G_{\varepsilon}+\partial_{x} F_{\varepsilon} \partial_{t} G_{\varepsilon}\right) \zeta \partial_{x} \zeta \mathrm{d} x \\
& -\frac{\mathrm{d}}{\mathrm{d} t} \int_{-L}^{L} F_{\varepsilon} G_{\varepsilon} \partial_{x}\left(\zeta \partial_{x} \zeta\right) \mathrm{d} x .
\end{aligned}
$$

We end up with the following formula for $J_{\varepsilon}$ :

$$
\begin{aligned}
J_{\varepsilon}=\frac{1}{2} \frac{\mathrm{d}}{\mathrm{d} t} \int_{-L}^{L}\left(F_{\varepsilon} g_{\varepsilon}+G_{\varepsilon} f_{\varepsilon}\right) \zeta^{2} \mathrm{~d} x & -\varepsilon^{2} \frac{\mathrm{d}}{\mathrm{d} t} \int_{-L}^{L} F_{\varepsilon} G_{\varepsilon} \partial_{x}\left(\zeta \partial_{x} \zeta\right) \mathrm{d} x \\
& -2 \varepsilon^{2} \int_{-L}^{L}\left(\partial_{t} F_{\varepsilon} \partial_{x} G_{\varepsilon}+\partial_{x} F_{\varepsilon} \partial_{t} G_{\varepsilon}\right) \zeta \partial_{x} \zeta \mathrm{d} x .
\end{aligned}
$$

After integration over $(0, T)$, it follows from (3.9)-(3.11) and the previous identity that

$$
\begin{aligned}
\frac{1}{2}(1+R) \| & f_{\varepsilon}(T) \zeta\left\|_{2}^{2}-\frac{1}{2}(1+R)\right\| f_{\varepsilon}(0) \zeta\left\|_{2}^{2}+\frac{1}{2} R\right\| g_{\varepsilon}(T) \zeta\left\|_{2}^{2}-\frac{1}{2} R\right\| g_{\varepsilon}(0) \zeta \|_{2}^{2} \\
& +\frac{1}{2} R \int_{-L}^{L}\left(F_{\varepsilon} g_{\varepsilon}+G_{\varepsilon} f_{\varepsilon}\right)(T) \zeta^{2} \mathrm{~d} x-\frac{1}{2} R \int_{-L}^{L}\left(F_{\varepsilon} g_{\varepsilon}+G_{\varepsilon} f_{\varepsilon}\right)(0) \zeta^{2} \mathrm{~d} x \\
& -R \varepsilon^{2} \int_{-L}^{L}\left(F_{\varepsilon} G_{\varepsilon}\right)(T) \partial_{x}\left(\zeta \partial_{x} \zeta\right) \mathrm{d} x+R \varepsilon^{2} \int_{-L}^{L}\left(F_{\varepsilon} G_{\varepsilon}\right)(0) \partial_{x}\left(\zeta \partial_{x} \zeta\right) \mathrm{d} x \\
& -2 R \varepsilon^{2} \int_{0}^{T} \int_{-L}^{L}\left(\partial_{t} F_{\varepsilon} \partial_{x} G_{\varepsilon}+\partial_{x} F_{\varepsilon} \partial_{t} G_{\varepsilon}\right) \zeta \partial_{x} \zeta \mathrm{d} x \mathrm{~d} s \\
= & -\int_{0}^{T} \int_{-L}^{L} \sqrt{f_{\varepsilon}} U_{\varepsilon} \partial_{x}\left[\left((1+R) f_{\varepsilon}+R G_{\varepsilon}\right) \zeta^{2}\right] \mathrm{d} x \mathrm{~d} s+\int_{0}^{T} I_{1, \varepsilon} \mathrm{d} s \\
& -R R_{\mu} \int_{0}^{T} \int_{-L}^{L} \sqrt{g_{\varepsilon}} V_{\varepsilon} \partial_{x}\left[\left(F_{\varepsilon}+g_{\varepsilon}\right) \zeta^{2}\right] \mathrm{d} x \mathrm{~d} s+\int_{0}^{T} I_{2, \varepsilon} \mathrm{d} s .
\end{aligned}
$$

Using Young's inequality we get

$$
\begin{aligned}
\frac{1}{2}(1+R)\left\|f_{\varepsilon}(T) \zeta\right\|_{2}^{2}+\frac{1}{2} R\left\|g_{\varepsilon}(T) \zeta\right\|_{2}^{2}+\frac{1}{2} R \int_{-L}^{L}\left(F_{\varepsilon} g_{\varepsilon}+G_{\varepsilon} f_{\varepsilon}\right)(T) \zeta^{2} \mathrm{~d} x \\
+K_{\varepsilon}(T)+\frac{1}{2} \int_{0}^{T} \int_{-L}^{L}\left[U_{\varepsilon}^{2}+R R_{\mu} V_{\varepsilon}^{2}\right] \zeta^{2} \mathrm{~d} x \mathrm{~d} s \\
\leqslant \frac{1}{2}(1+R)\left\|f_{\varepsilon}(0) \zeta\right\|_{2}^{2}+\frac{1}{2} R\left\|g_{\varepsilon}(0) \zeta\right\|_{2}^{2}+\frac{1}{2} R \int_{-L}^{L}\left(F_{\varepsilon} g_{\varepsilon}+G_{\varepsilon} f_{\varepsilon}\right)(0) \zeta^{2} \mathrm{~d} x \\
+2 \int_{0}^{T} \int_{-L}^{L}\left[f_{\varepsilon}\left|(1+R) f_{\varepsilon}+R G_{\varepsilon}\right|^{2}+R R_{\mu} g_{\varepsilon}\left|F_{\varepsilon}+g_{\varepsilon}\right|^{2}\right]\left|\partial_{x} \zeta\right|^{2} \mathrm{~d} x \mathrm{~d} s
\end{aligned}
$$


with

$$
\begin{aligned}
K_{\varepsilon}(T):= & -R \varepsilon^{2} \int_{-L}^{L}\left(F_{\varepsilon} G_{\varepsilon}\right)(T) \partial_{x}\left(\zeta \partial_{x} \zeta\right) \mathrm{d} x+R \varepsilon^{2} \int_{-L}^{L}\left(F_{\varepsilon} G_{\varepsilon}\right)(0) \partial_{x}\left(\zeta \partial_{x} \zeta\right) \mathrm{d} x \\
& -2 R \varepsilon^{2} \int_{0}^{T} \int_{-L}^{L}\left(\partial_{t} F_{\varepsilon} \partial_{x} G_{\varepsilon}+\partial_{x} F_{\varepsilon} \partial_{t} G_{\varepsilon}\right) \zeta \partial_{x} \zeta \mathrm{d} x \mathrm{~d} s-\int_{0}^{T}\left(I_{1, \varepsilon}+I_{2, \varepsilon}\right) \mathrm{d} s .
\end{aligned}
$$

According to [6], the convergences of $\left(f_{\varepsilon_{k}}\right)_{k}$ and $\left(g_{\varepsilon_{k}}\right)_{k}$ towards $f$ and $g$ actually take place in stronger topologies than stated in proposition 3.4. In fact, for all $T>0$,

$$
f_{\varepsilon_{k}} \rightarrow f, \quad F_{\varepsilon_{k}} \rightarrow f, \quad g_{\varepsilon_{k}} \rightarrow g, \quad G_{\varepsilon_{k}} \rightarrow g \quad \text { in } L_{2}(0, T ; C([-L, L])),
$$

$f_{\varepsilon_{k}}(0) \rightarrow f_{0}, \quad F_{\varepsilon_{k}}(0) \rightarrow f_{0}, \quad g_{\varepsilon_{k}}(0) \rightarrow g_{0}, \quad G_{\varepsilon_{k}}(0) \rightarrow g_{0} \quad$ in $L_{2}((-L, L))$,

and

$$
\begin{array}{ll}
U_{\varepsilon_{k}} \rightarrow U:=\sqrt{f}\left((1+R) \partial_{x} f+R \partial_{x} g\right) & \text { in } L_{2}((0, T) \times(-L, L)), \\
V_{\varepsilon_{k}} \rightarrow V:=\sqrt{g}\left(\partial_{x} f+\partial_{x} g\right) & \text { in } L_{2}((0, T) \times(-L, L)) .
\end{array}
$$

Furthermore, it follows from [6, lemmas 2.3 and 2.5] that

$\left(\left(f_{\varepsilon_{k}}, g_{\varepsilon_{k}}, F_{\varepsilon_{k}}, G_{\varepsilon_{k}}\right)\right)_{k}$ are bounded in $L_{\infty}\left(0, T ; L_{2}(-L, L)\right) \cap L_{2}\left(0, T ; H^{1}(-L, L)\right)$.

We also infer from (3.13) that

$\lim _{k \rightarrow \infty}\left\{\left\|\left(f_{\varepsilon_{k}}-f\right)(T)\right\|_{2}+\left\|\left(F_{\varepsilon_{k}}-f\right)(T)\right\|_{2}+\left\|\left(g_{\varepsilon_{k}}-g\right)(T)\right\|_{2}+\left\|\left(G_{\varepsilon_{k}}-g\right)(T)\right\|_{2}\right\}=0$

for almost all $T>0$. We may then take $\varepsilon=\varepsilon_{k}$ in (3.12) and let $k \rightarrow \infty$ to deduce from (3.13)-(3.18) that, for almost all $T>0$,

$$
\begin{aligned}
& \frac{1+R}{2}\|f(T) \zeta\|_{2}^{2}+\frac{1}{2} R\|g(T) \zeta\|_{2}^{2}+R \int_{-L}^{L}(f g)(T) \zeta^{2} \mathrm{~d} x \\
& \quad+\frac{1}{2} \int_{0}^{T} \int_{-L}^{L}\left[U^{2}+R R_{\mu} V^{2}\right] \zeta^{2} \mathrm{~d} x \mathrm{~d} s \\
& \leqslant \frac{1+R}{2}\left\|f_{0} \zeta\right\|_{2}^{2}+\frac{1}{2} R\left\|g_{0} \zeta\right\|_{2}^{2}+R \int_{-L}^{L} f_{0} g_{0} \zeta^{2} \mathrm{~d} x \\
& \quad+2 \int_{0}^{T} \int_{-L}^{L}\left[f|(1+R) f+R g|^{2}+R R_{\mu} g|f+g|^{2}\right]\left|\partial_{x} \zeta\right|^{2} \mathrm{~d} x \mathrm{~d} s
\end{aligned}
$$

provided we establish that

$$
\lim _{k \rightarrow \infty} K_{\varepsilon_{k}}(T)=0
$$




\subsubsection{The term $K_{\varepsilon}(T)$}

It remains to prove (3.20) and actually identify the behaviour of $K_{\varepsilon}(T)$ as $\varepsilon \rightarrow 0$. Owing to (3.14) and (3.18), it is clear that

$$
\begin{aligned}
& \lim _{k \rightarrow \infty} R \varepsilon_{k}^{2} \int_{-L}^{L}\left(F_{\varepsilon_{k}} G_{\varepsilon_{k}}\right)(T) \partial_{x}\left(\zeta \partial_{x} \zeta\right) \mathrm{d} x \\
&=\lim _{k \rightarrow \infty} R \varepsilon_{k}^{2} \int_{-L}^{L}\left(F_{\varepsilon_{k}} G_{\varepsilon_{k}}\right)(0) \partial_{x}\left(\zeta \partial_{x} \zeta\right) \mathrm{d} x=0
\end{aligned}
$$

for almost all $T>0$. Next, it readily follows from (3.17) that

$$
\lim _{\varepsilon \rightarrow 0} \int_{0}^{T} I_{1, \varepsilon} \mathrm{d} t=\lim _{\varepsilon \rightarrow 0} \int_{0}^{T} I_{2, \varepsilon} \mathrm{d} t=0 .
$$

Finally, since

$$
\partial_{t} f_{\varepsilon}=\partial_{x}\left(\sqrt{f_{\varepsilon}} U_{\varepsilon}-R \varepsilon \partial_{x} G_{\varepsilon}\right)
$$

the boundedness $(3.17)$ of $\left(U_{\varepsilon}\right)_{\varepsilon}$ in $L_{2}((0, T) \times(-L, L))$, and the boundedness of $\left(f_{\varepsilon}\right)_{\varepsilon}$ in $L_{\infty}\left(0, T ; L_{2}(-L, L)\right)$ imply the boundedness of $\left(\sqrt{f_{\varepsilon}} U_{\varepsilon}-R \varepsilon \partial_{x} G_{\varepsilon}\right)_{\varepsilon}$ in $L_{2}\left(0, T ; L_{4 / 3}(-L, L)\right)$. As a consequence, the sequence $\left(\partial_{t} f_{\varepsilon}\right)_{\varepsilon}$ is bounded in $L_{2}\left(0, T ;\left(W_{4}^{1}(-L, L)\right)^{\prime}\right)$, and so, by a similar argument, is $\left(\partial_{t} g_{\varepsilon}\right)_{\varepsilon}$. Owing to the properties of $\left(1-\varepsilon^{2} \partial_{x}^{2}\right)^{-1}$, we conclude that

$$
\left(\partial_{t} F_{\varepsilon}\right)_{\varepsilon},\left(\partial_{t} G_{\varepsilon}\right)_{\varepsilon} \text { are bounded in } L_{2}\left(0, T ;\left(W_{4}^{1}\right)^{\prime}\right)
$$

(see [6, lemma 3.1] for a similar result). Now, since $\zeta \in C_{0}^{\infty}((-L, L))$ and $W_{4}^{1}(-L, L)$ is an algebra, we infer from (3.23) that

$$
\begin{aligned}
2 R \varepsilon^{2} \mid & \int_{0}^{T} \int_{-L}^{L}\left(\partial_{t} F_{\varepsilon} \partial_{x} G_{\varepsilon}+\partial_{x} F_{\varepsilon} \partial_{t} G_{\varepsilon}\right) \zeta \partial_{x} \zeta \mathrm{d} x \mathrm{~d} s \mid \\
& \leqslant C \varepsilon^{2} \int_{0}^{T}\left(\left\|\partial_{t} F_{\varepsilon}\right\|_{\left(W_{4}^{1}\right)^{\prime}}\left\|\partial_{x} G_{\varepsilon}\right\|_{W_{4}^{1}}+\left\|\partial_{t} G_{\varepsilon}\right\|_{\left(W_{4}^{1}\right)^{\prime}}\left\|\partial_{x} F_{\varepsilon}\right\|_{W_{4}^{1}}\right)\left\|\zeta \partial_{x} \zeta\right\|_{W_{4}^{1}} \mathrm{~d} s \\
& \leqslant C(\zeta, T) \varepsilon^{2}\left[\int_{0}^{T}\left(\left\|\partial_{x} G_{\varepsilon}\right\|_{W_{4}^{1}}^{2}+\left\|\partial_{x} F_{\varepsilon}\right\|_{W_{4}^{1}}^{2}\right) \mathrm{d} s\right]^{1 / 2}
\end{aligned}
$$

Now, owing to (3.1), for almost all $t \in(0, T)$ the function $\partial_{x} F_{\varepsilon}(t)$ solves

$$
\partial_{x} F_{\varepsilon}-\varepsilon^{2} \partial_{x}^{2} \partial_{x} F_{\varepsilon}=\partial_{x} f_{\varepsilon} \quad \text { in }(-L, L), \quad \partial_{x} F_{\varepsilon}( \pm L)=0,
$$

which implies that

$$
\left\|\partial_{x} F_{\varepsilon}\right\|_{2}^{2}+\varepsilon^{2}\left\|\partial_{x}^{2} F_{\varepsilon}\right\|_{2}^{2}+\varepsilon^{4}\left\|\partial_{x}^{3} F_{\varepsilon}\right\|_{2}^{2} \leqslant C\left\|\partial_{x} f_{\varepsilon}\right\|_{2}^{2} .
$$

These estimates, along with the Gagliardo-Nirenberg inequality [14, theorem 1], give

$$
\begin{aligned}
\left\|\partial_{x} F_{\varepsilon}\right\|_{W_{4}^{1}} & \leqslant C\left(\left\|\partial_{x} F_{\varepsilon}\right\|_{4}+\left\|\partial_{x}^{2} F_{\varepsilon}\right\|_{4}\right) \\
& \leqslant C\left(\left\|\partial_{x}^{2} F_{\varepsilon}\right\|_{2}^{1 / 4}\left\|\partial_{x} F_{\varepsilon}\right\|_{2}^{3 / 4}+\left\|\partial_{x}^{3} F_{\varepsilon}\right\|_{2}^{1 / 4}\left\|\partial_{x}^{2} F_{\varepsilon}\right\|_{2}^{3 / 4}+\left\|\partial_{x}^{2} F_{\varepsilon}\right\|_{2}\right) \\
& \leqslant C \varepsilon^{-5 / 4}\left\|\partial_{x} f_{\varepsilon}\right\|_{2} .
\end{aligned}
$$


As a similar estimate is valid for $\left\|\partial_{x} G_{\varepsilon}\right\|_{W_{4}^{1}}$ with $\left\|\partial_{x} g_{\varepsilon}\right\|_{2}$ instead of $\left\|\partial_{x} f_{\varepsilon}\right\|_{2}$, we deduce from (3.17) and (3.24) that

$$
\begin{gathered}
2 R \varepsilon^{2}\left|\int_{0}^{T} \int_{-L}^{L}\left(\partial_{t} F_{\varepsilon} \partial_{x} G_{\varepsilon}+\partial_{x} F_{\varepsilon} \partial_{t} G_{\varepsilon}\right) \zeta \partial_{x} \zeta \mathrm{d} x \mathrm{~d} s\right| \\
\leqslant C(\zeta, T) \varepsilon^{3 / 4}\left[\int_{0}^{T}\left(\left\|\partial_{x} f_{\varepsilon}\right\|_{2}^{2}+\left\|\partial_{x} g_{\varepsilon}\right\|_{2}^{2}\right) \mathrm{d} s\right]^{1 / 2} \\
\leqslant C(\zeta, T) \varepsilon^{3 / 4}
\end{gathered}
$$

Combining (3.21), (3.22) and (3.25) gives the claim (3.20) and completes the proof of (3.8) for $\zeta \in C_{0}^{\infty}(\mathbb{R})$, its validity for all $T>0$ being obtained by a lower semicontinuity argument. According to the regularity of $(f, g)$, the extension of lemma 3.5 to all functions $\zeta \in W_{4}^{1}((-L, L))$ follows by a density argument.

\subsection{The limit $L \rightarrow \infty$}

For each positive $L$, we denote the couple found in proposition 3.4 by $\left(f^{L}, g^{L}\right)$. The family $\left(\left(f^{L}, g^{L}\right)\right)_{L}$ satisfies the same bounds as the family $\left(\left(f_{\varepsilon}, g_{\varepsilon}\right)\right)_{\varepsilon}$, so that taking the limit as $L \rightarrow \infty$ may be done as $\varepsilon \rightarrow 0$, the only difference being the unboundedness of the domain that one has to cope with. To this end we derive the following lemma, which controls the behaviour of $\left(f^{L}, g^{L}\right)$ at infinity.

LEMMA 3.6. It holds that

$$
\int_{-L / 2}^{L / 2}\left(f^{L}+\frac{R}{R_{\mu}} g^{L}\right)(T) x^{2} \mathrm{~d} x \leqslant \int_{-L}^{L}\left(f_{0}+\frac{R}{R_{\mu}} g_{0}\right) x^{2} \mathrm{~d} x+T \mathcal{E}\left(f_{0}, g_{0}\right)
$$

for all $T>0$.

Proof. We define the function

$$
\Phi(x)= \begin{cases}-2 L x-x^{2}-\frac{3}{4} L^{2}, & -L \leqslant x \leqslant-\frac{1}{2} L, \\ x^{2}, & -\frac{1}{2} L \leqslant x \leqslant \frac{1}{2} L, \\ 2 L x-x^{2}-\frac{3}{4} L^{2}, & \frac{1}{2} L \leqslant x \leqslant L .\end{cases}
$$

We take $\xi=\Phi$ in (3.6) and $\xi=R \Phi / R_{\mu}$ in (3.7). Then, using integration by parts and the bound $\Phi^{\prime \prime} \leqslant 2$, we obtain

$$
\begin{aligned}
\int_{-L}^{L}\left(f^{L}+\frac{R}{R_{\mu}} g^{L}\right)(T) \Phi \mathrm{d} x & =\int_{-L}^{L}\left(f_{0}+\frac{R}{R_{\mu}} g_{0}\right) \Phi \mathrm{d} x \\
& +\frac{1}{2} \int_{0}^{T} \int_{-L}^{L}\left(\left(f^{L}\right)^{2}+R\left(f^{L}+g^{L}\right)^{2}\right) \Phi^{\prime \prime} \mathrm{d} x \mathrm{~d} t \\
& \leqslant \int_{-L}^{L}\left(f_{0}+\frac{R}{R_{\mu}} g_{0}\right) \Phi \mathrm{d} x+T \mathcal{E}\left(f_{0}, g_{0}\right) .
\end{aligned}
$$

In addition,

$$
x^{2} \mathbf{1}_{[-L / 2, L / 2]} \leqslant \Phi(x) \leqslant x^{2} \text { for } x \in[-L, L],
$$

and the claim follows. 
Using lemma 3.6 we may argue as in the proof of proposition 3.4 (see [6]), to take the limit as $L \rightarrow \infty$ and complete the proof of theorem 3.1. In particular, we use lemma 3.6 to establish the entropy inequality in theorem 3.1(b) as well as the conservation of mass in theorem 3.1.

\section{Acknowledgements}

This work was partly supported by the French-German Procope project 30718ZG and by the Agence Nationale de la Recherche through Grant no. ANR-13-JS010007-01 (GeoPor project).

\section{References}

1 H. Amann. Nonhomogeneous linear and quasilinear elliptic and parabolic boundary value problems. In Function spaces, differential operators and nonlinear analysis. Teubner-Texte zur Mathematik, vol. 133, pp. 9-126 (Stuttgart: Teubner, 1993).

2 S. N. Antontsev, J. I. Díaz and S. Shmarev. Energy methods for free boundary problems. Progress in Nonlinear Differential Equations and Their Applications, vol. 48 (Boston, MA: Birkhäuser, 2002).

3 F. Bernis. Finite speed of propagation and continuity of the interface for thin viscous flows. Adv. Diff. Eqns 1 (1996), 337-368.

4 R. Dal Passo, L. Giacomelli and G. Grün. Waiting time phenomena for degenerate parabolic equations: a unifying approach. In Geometric analysis and nonlinear partial differential equations, pp. 637-648 (Berlin: Springer, 2003).

5 J. I. Díaz, G. Galiano and A. Jüngel. On a quasilinear degenerate system arising in semiconductor theory. II. Localization of vacuum solutions. Nonlin. Analysis 36 (1999), 569-594.

6 J. Escher, Ph. Laurençot and B.-V. Matioc. Existence and stability of weak solutions for a degenerate parabolic system modelling two-phase flows in porous media. Annales Inst. $H$. Poincaré Analyse Non Linéaire 28 (2011), 583-598.

7 J. Escher, A.-V. Matioc and B.-V. Matioc. Modelling and analysis of the Muskat problem for thin fluid layers. J. Math. Fluid Mech. 14 (2012), 267-277.

8 J. Fischer. Advection-driven support shrinking in a chemotaxis model with degenerate mobility. SIAM J. Math. Analysis 45 (2013), 1585-1615.

9 M. Jazar and R. Monneau. Derivation of seawater intrusion models by formal asymptotics. SIAM J. Appl. Math. 74 (2014), 1152-1173.

10 Ph. Laurençot and B.-V. Matioc. A gradient flow approach to a thin film approximation of the Muskat problem. Calc. Var. PDEs 47 (2013), 319-341.

11 Ph. Laurençot and B.-V. Matioc. A thin film approximation of the Muskat problem with gravity and capillary forces. J. Math. Soc. Jpn 66 (2014), 1043-1071.

12 Ph. Laurençot and B.-V. Matioc. Self-similarity in a thin film Muskat problem. Preprint, 2014. (Available at https://arxiv.org/abs/1409.7329v1.)

13 M. Muskat. Two fluid systems in porous media: the encroachment of water into an oil sand. Physics 5 (1934), 250-264.

14 L. Nirenberg. An extended interpolation inequality. Annali Scuola Norm. Sup. Pisa 20 (1966), 733-737.

15 A. E. Shishkov. Estimates for the rate of propagation of perturbations in quasilinear degenerate higher-order parabolic equations in divergence form. Ukrä̈n. Mat. Zh. 44 (1992), $1451-1456$.

16 J. L. Vázquez. The porous medium equation: mathematical theory. Oxford Mathematical Monographs (Oxford: Clarendon Press, 2007). 